\title{
Determination of energy and duration of picosecond light pulses by bleaching of dyes
}

\author{
G. GRÖNNINGER, A. PENZKOFER \\ Naturwissenschaftliche Fakultät I/ - Physik, Universität Regensburg, 8400 Regensburg, \\ Federal Republic of Germany
}

Received 15 August 1983

The transmission of a light pulse through a dye solution is determined by its energy density if the pulse duration is short compared to the absorption recovery time. The energy transmission measurement allows the determination of the pulse energy. Simultaneous measurement of energy transmission and input pulse peak intensity makes it possible to calculate the pulse duration.

\section{Introduction}

The interaction of intense picosecond light pulses with absorbing dye solutions promotes an appreciable number of molecules to an excited state and reduces the ground state absorption. For pulse durations $\Delta t_{L}$ short compared to the absorption recovery time $\tau_{\mathrm{F}}$ the number of excited molecules becomes equal to the number of absorbed photons if the excited state absorption is weak. A measurement of the pulse transmission through a dye sample allows one to determine the pulse energy, $E$. Since the pulse energy is proportional to the peak pulse intensity and to the pulse duration, a measurement of energy transmission $T_{E}$ and input peak intensity $I_{0 L}$ makes it possible to calculate the pulse duration $\Delta t_{L}$ for $\Delta t_{L}<\tau_{\mathrm{F}}$.

In this article we calculate plots of $T_{E}(E)$ and $T_{E}\left(I_{0 L}, \Delta t_{L}\right)$ for energy and pulse duration detection, respectively. Curves are presented for the interaction of dyes with picosecond light pulses of a ruby laser (fundamental and second harmonic) and an $\mathrm{Nd}$-glass laser (fundamental, second, third, and fourth harmonic). The calculations are verified experimentally for a mode-locked ruby laser. The energy detection is applied to calibrate photodetectors to absolute energy values. The measurement of $T_{E}$ and $I_{0 L}$ gives an inexpensive possibility of picosecond pulse duration measurement.

\section{Theory}

The dye solutions are described by a realistic four level system as depicted in Fig. 1 [1]. The laser light excites molecules from the singlet ground state $S_{0}$ to a Franck-Condon level 2 in the $S_{1}$ band (absorption cross-section $\sigma_{L}$ ). From there the molecules relax quickly to a temporal equilibrium position 3 in the $S_{1}$ state (relaxation time $\tau_{\mathrm{FC}}=0.7$ ps assumed in our calculations [2, 3]). Excited state absorption (cross-section $\sigma_{\mathrm{ex}}$ ) may promote molecules from $S_{1}$ to higher lying states $S_{n}$. A fast relaxation from $S_{n}$ to $S_{1}$ is assumed in the calculations $\left(\tau_{\mathrm{ex}}=10^{-13} \mathrm{~s}[4-6]\right)$. The molecules in the $S_{1}$ band relax to the ground state with a time constant $\tau_{\mathrm{F}}$. Depopulation of the $S_{1}$ state by amplified spontaneous emission is negligible for the applied dye concentrations (small signal transmission $T_{0}=0.01$ ) [1,5] and is not included in the level scheme and the calculations. Triplet states are neglected since dyes with small intersystem-crossing rates are selected.

The dynamics of interaction of dye molecules with laser light is governed by the following differential equation system

$$
\frac{\partial N_{1}(\theta)}{\partial t^{\prime}}=-\frac{I_{L}}{h \nu_{L}} 3 \sigma_{L} \cos ^{2} \theta\left[N_{1}(\theta)-N_{2}(\theta)\right]+\frac{N_{2}(\theta)+N_{3}(\theta)}{\tau_{\mathbf{F}}}-\frac{N_{1}(\theta)-\bar{N}_{1}}{\tau_{\text {or }}}
$$




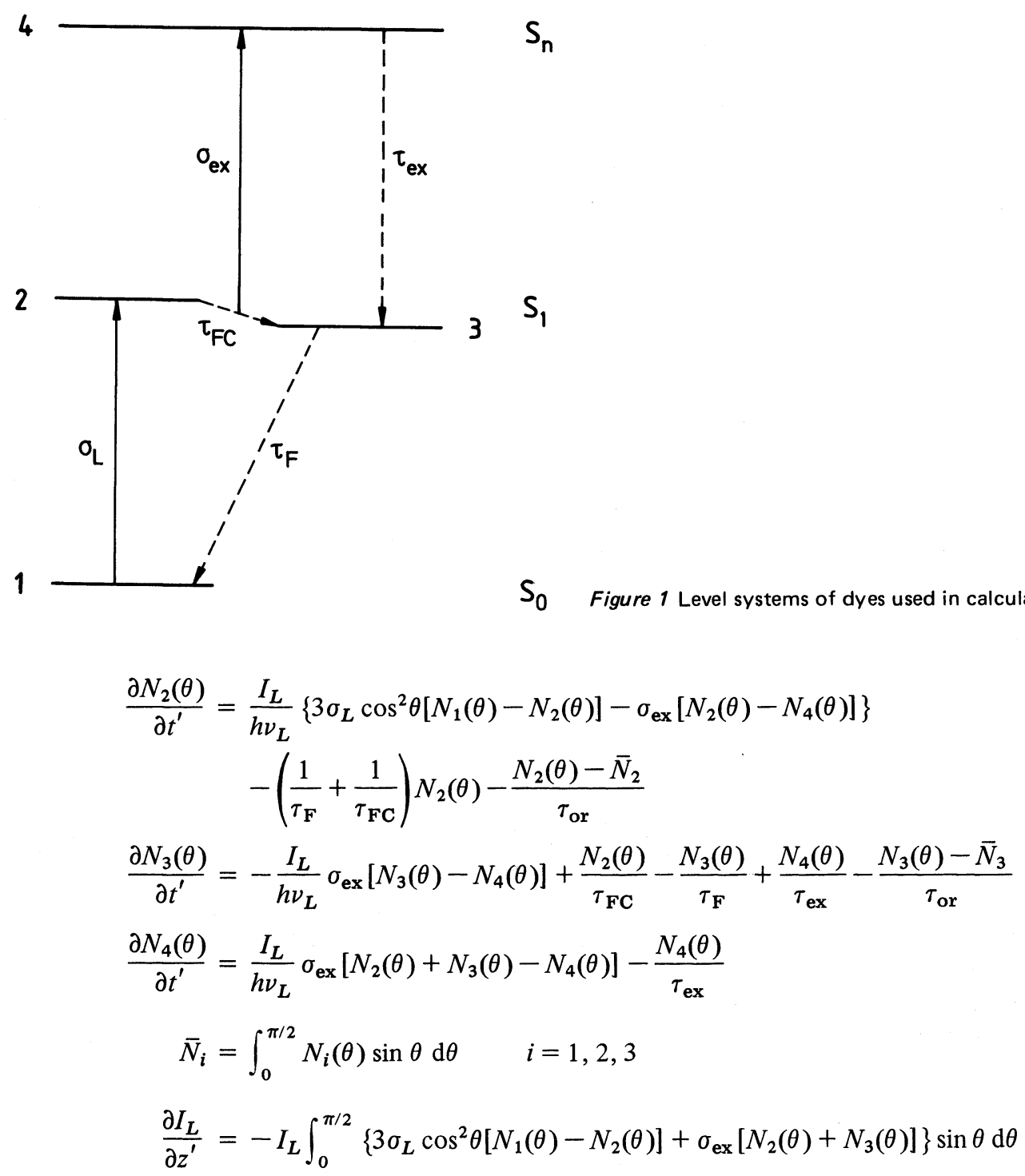

The transformation $t^{\prime}=t-c z / \eta$ and $z^{\prime}=z$ is used ( $c$ is vacuum light velocity; $\eta$ is refractive index). The initial conditions are $N_{1}\left(\theta, t^{\prime}=-\infty, z^{\prime}, r\right)=N_{0}, N_{2}\left(\theta, t^{\prime}=-\infty, z^{\prime}, r\right)=N_{3}\left(\theta, t^{\prime}=-\infty, z^{\prime}, r\right)=$ $N_{4}\left(\theta, t^{\prime}=-\infty, z^{\prime}, r\right)=0$ and $I_{L}\left(t^{\prime}, z^{\prime}=0, r\right)=I_{0 L} f\left(t^{\prime} / t_{0}\right) g\left(r / r_{0}\right) . N_{0}$ is the total density of dissolved dye molecules. $f\left(t^{\prime} / t_{0}\right)$ and $g\left(r / r_{0}\right)$ are the temporal and spatial input pulse shapes, respectively.

The absorption anisotropy of electric dipole interaction is taken into account for the ground state absorption $\left[\sigma_{L}(\theta)=3 \sigma_{L} \cos ^{2} \theta\right.$ where $\theta$ is the angle between the transition dipole moment of molecules and the direction of the electric field of linearly polarized laser light]. The excited state absorption is included by an effective isotropic absorption cross-section $\sigma_{\mathbf{e x}}$ (orientation of excited state transition dipole moments [7] is unknown). $\tau_{\text {or }}$ describes the molecular reorientation of the transition dipole moments.

Equations 1-6 are solved numerically. The intensity transmission is given by $T_{I}\left(t^{\prime}, r\right)=I_{L}\left(t^{\prime}, l, r\right) /$ $I_{L}\left(t^{\prime}, 0, r\right)$. The transmission of the energy density $\epsilon$ is $T_{\epsilon}(r)=\epsilon(l, r) / \epsilon(0, r)=\int_{-\infty}^{\infty} I_{L}\left(t^{\prime}, l, r\right) \mathrm{d} t^{\prime} /$ $\int_{-\infty}^{\infty} I_{L}\left(t^{\prime}, 0, r\right) \mathrm{d} t^{\prime}$. Finally the energy transmission is $T_{E}=E(l) / E(0)=\int_{0}^{\infty} \epsilon(l, r) r \mathrm{~d} r / \int_{0}^{\infty} \epsilon(0, r) r \mathrm{~d} r$.

For pulse durations $\Delta t_{L}(\mathrm{FWHM})$ in the region $20 \tau_{\mathrm{FC}}<\Delta t_{L}<\tau_{\text {or }} / 2$ Equations 1-6 may be approxi- 

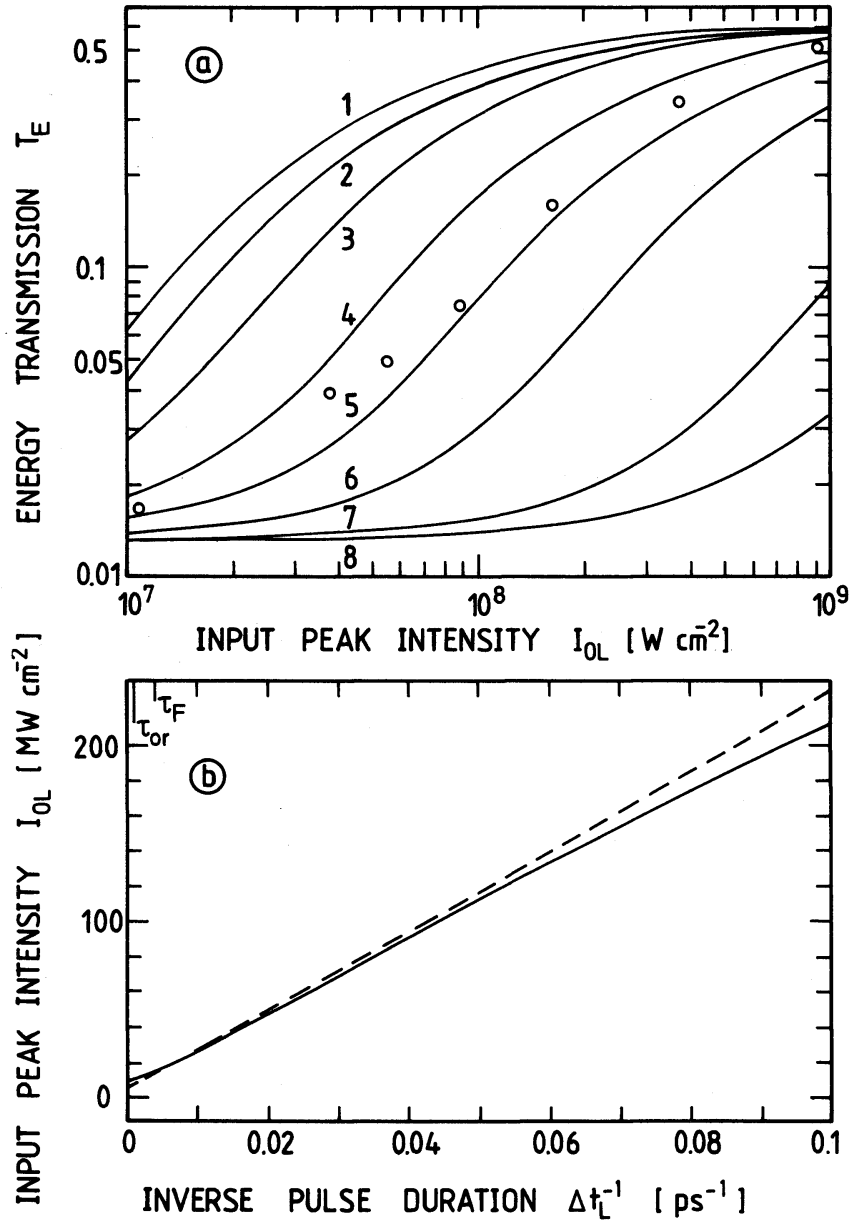

Figure 2 (a) Energy transmission against input peak pulse intensity. Curves apply to transmission of ruby laser pulses of temporal and spatial Gaussian shape through DDI dissolved in glycerol. Small signal transmission $T_{0}=0.013$ (see Table I for dye parameters). The pulse durations $\Delta t_{L}$ are (1) $520 \mathrm{ps,}$

(2) $260 \mathrm{ps},(3) 130 \mathrm{ps},(4) 50 \mathrm{ps},(5) 25 \mathrm{ps}$, (6) $10 \mathrm{ps},(7) 2 \mathrm{ps}$ and (8) $0.7 \mathrm{ps}$. Circles are experimental points for $\Delta t_{L}=30 \pm 3$ ps.

(b) Input peak intensity against inverse pulse duration for $T_{E}=0.07$. Solid curve is for data as in 2a (Equations 1-6). Dashed curve is linearized solution (Equations 7-10) responsible for $\tau_{\mathrm{FC}}=0$ and $\tau_{\mathrm{or}}=\infty$.

mated by setting $\tau_{\mathrm{FC}}=0$ and $\tau_{\text {or }}=\infty$ (deviation about $5 \%$ at upper and lower limits). The resulting equations are

$$
\begin{aligned}
\frac{\partial N_{1}(\theta)}{\partial t^{\prime}} & =-\frac{I_{L}}{h \nu_{L}} 3 \sigma_{L} \cos ^{2} \theta N_{1}(\theta)+\frac{N_{3}(\theta)}{\tau_{\mathrm{F}}} \\
\frac{\partial N_{3}(\theta)}{\partial t^{\prime}} & =\frac{I_{L}}{h \nu_{L}}\left\{3 \sigma_{L} \cos ^{2} \theta N_{1}(\theta)-\sigma_{\mathrm{ex}}\left[N_{3}(\theta)-N_{4}(\theta)\right]\right\}-\frac{N_{3}(\theta)}{\tau_{\mathrm{F}}}+\frac{N_{4}(\theta)}{\tau_{\mathrm{ex}}} \\
\frac{\partial N_{4}(\theta)}{\partial t^{\prime}} & =\frac{I_{L}}{h \nu_{L}} \sigma_{\mathrm{ex}}\left[N_{3}(\theta)-N_{4}(\theta)\right]-\frac{N_{4}(\theta)}{\tau_{\mathrm{ex}}} \\
\frac{\partial I_{L}}{\partial t^{\prime}} & =-I_{L} \int_{0}^{\pi / 2}\left[3 \sigma_{L} \cos ^{2} \theta N_{1}(\theta)+\sigma_{\mathrm{ex}} N_{3}(\theta)\right] \sin \theta \mathrm{d} \theta
\end{aligned}
$$

In Fig. 2a $T_{E}$ against $I_{0 L}$ is plotted for various pulse durations $\Delta t_{L}$ (FWHM). A Gaussian temporal and spatial pulse shape is assumed $\left[\Delta t_{L}=2(\ln 2)^{1 / 2} t_{0}\right]$. The curves are calculated using Equations $1-6$. The used parameters apply to the passage of ruby laser pulses through DDI dissolved in glycerol $\left(T_{0}=0.013\right.$, for other data see Table I). For $\Delta t_{L}>\tau_{\mathrm{F}}$ the $T_{E}\left(I_{0 L}\right)$ curves change only slightly with pulse duration. The detection of $\Delta t_{L}$ from $T_{E}\left(I_{0 L}\right)$ measurement is limited to $\Delta t_{L} \lesssim \tau_{F} / 2$.

The curves of Fig. 2a are redrawn in Fig. 2b to depict the dependence of $I_{0 L}$ on $\Delta t_{L}^{-1}$ for a fixed energy transmission of $T_{E}=0.07$ (solid curve). The dashed curve is obtained by solving the reduced 
Equations 7-10. In this case a linear dependence of $I_{0 L}$ on $\Delta t_{L}^{-1}$ is found, i.e. $I_{0 L}\left(\Delta t_{L}^{-1}\right)=\bar{I}_{0 L}(1+$ $\left.\kappa_{\mathrm{F}} \tau_{\mathrm{F}} \Delta t_{L}^{-1}\right) . \bar{I}_{0 L}$ is the peak intensity value at $\Delta t_{L}^{-1}=0 . \kappa_{\mathrm{F}}$ is a constant near to 1 which depends slightly on the temporal pulse shape and is independent of $T_{E}$ and $\sigma_{\mathrm{ex}}\left(\kappa_{\mathrm{F}}=1.43\right.$ for Gaussian shape, $\kappa_{\mathrm{F}}=1.94$ for rectangular temporal shape).

The linear dependence of $I_{0 L}$ on $\Delta t_{L}^{-1}$ for $\tau_{\mathrm{FC}}=0$ and $\tau_{\mathrm{or}} \rightarrow \infty$ (dashed curve in Fig. 2b) allows one to determine an apparent duration $\Delta t_{L, a}$ from a measurement of $T_{E}$ and $I_{0 L}$ if a calibration curve $T_{E}\left(I_{0 L}^{\prime}, \Delta t_{L}^{\prime}\right)$ for $\tau_{\mathrm{FC}}=0$ and $\tau_{\text {or }} \rightarrow \infty$ is known. At a fixed $T_{E}$ value the following relation between $\Delta t_{L, a}$ and $\Delta t_{L}^{\prime}$ is found by solving $I_{0 L}=\bar{I}_{0 L}\left(1+\kappa_{F} \tau_{F} / \Delta t_{L, a}\right)$ and $I_{0 L}^{\prime}=\bar{I}_{0 L}\left(1+\kappa_{F} \tau_{F} / \Delta t_{L}^{\prime}\right)$ to $\Delta t_{L, a}$ :

$$
\Delta t_{L, a}=\Delta t_{L}^{\prime} \frac{I_{0 L}^{\prime}}{I_{0 L}}\left[\left(1+\frac{\Delta t_{L}^{\prime}}{\kappa_{\mathrm{F}} \tau_{\mathrm{F}}}\right)\left(1-\frac{I_{0 L}^{\prime} / I_{0 L}}{1+\kappa_{\mathrm{F}} \tau_{\mathrm{F}} / \Delta t_{L}^{\prime}}\right)\right]^{-1}
$$

In the region $20 \tau_{\mathrm{FC}}<\Delta t_{L}<\tau_{\text {or }} / 2$ the $I_{0 L}\left(\Delta t_{L}^{-1}\right)$ dependence (solid curve of Fig. $2 \mathrm{~b}$ ) is well approximated by the linearized situation (dashed curve of Fig. $2 \mathrm{~b}$ ) and the apparent duration $\Delta t_{L, a}$ is equal to the true pulse duration $\Delta t_{L}$.

For pulse durations $\Delta t_{L} \lesssim 20 \tau_{\text {FC }}$ the population of the Franck-Condon level 2 is no longer negligible and the input peak intensity necessary to bleach the dye to transmission $T_{E}$ is reduced. For experimental situations obeying the conditions $\Delta t_{L}<\tau_{F} / 2$ and $\Delta t_{L} \gtrsim \tau_{\text {or }} / 2$ the reorientation of molecules within the pulse duration causes additional molecules to enter the strongly absorbing direction (small angle $\theta$ ) and the peak pulse intensity necessary to bleach the dye to $T_{E}$ increases. In the regions $\Delta t_{L} \lesssim 20 \tau_{\mathrm{FC}}$ (but $\Delta t_{L}>$ phase relaxation time $T_{2}$ ) and $\Delta t_{L}>\tau_{\text {or }} / 2$ (with $\Delta t_{L}<\tau_{F} / 2$ ) the true pulse duration may be obtained from the apparent duration $\Delta t_{L, a}$ (Equation 11) by multiplying with a correction factor $F_{t}$ which is plotted in Fig. $3 \mathrm{~b}$ for temporal Gaussian pulse shapes. $F_{t}\left(\Delta t_{L, a} / \tau_{\mathrm{FC}}\right)$ (lower curve) is independent of $T_{E}$ and $\sigma_{\text {ex }} \cdot F_{t}\left(\Delta t_{L, a} / \tau_{\text {or }}\right)$ (upper curve) is also practically independent of $T_{E}$. For $\Delta t_{L} / \tau_{\text {or }} \rightarrow \infty$ the ratio $F_{t}=\Delta t_{L} / \Delta t_{L, a}$ approaches the value of approximately 1.8 .

The $T_{E}\left(I_{0 L}, \Delta t_{L}\right)$ curves of Fig. 2a are redrawn in Fig. 4 a to present the dependence of $T_{E}$ on normalized pulse energy $E /\left(\pi r_{0}^{2}\right)\left[E=2 \pi I_{0 L} \int_{0}^{\infty} \int_{-\infty}^{\infty} f\left(t^{\prime} / t_{0}\right) g\left(r / r_{0}\right) r \mathrm{~d} t^{\prime} \mathrm{d} r\right]$. A temporal and spatial Gaussian shape is assumed. In Fig. $4 \mathrm{~b}$ the normalized pulse energy is plotted against pulse duration for a fixed energy transmission of $T_{E}=0.07$. The solid curve (Equations 1-6) is well approached by the dashed curve (Equations 7-10, $\tau_{\mathrm{FC}}=0, \tau_{\mathrm{or}}=\infty$ ) in the time region $20 \tau_{\mathrm{FC}}<\Delta t_{L}<\tau_{\text {or }} / 2$. The dashed curve obeys the equation $E_{a}\left(\Delta t_{L}\right)=E_{0}\left[1+\Delta t_{L} /\left(\kappa_{F} \tau_{F}\right)\right]$. In this linear region the pulse energy $E_{a}\left(\Delta t_{L}\right)$ belonging to $T_{E}$ is related to $E^{\prime}\left(\Delta t_{L}^{\prime}\right)$ at the same transmission $T_{E}$ by

$$
E_{a}\left(\Delta t_{L}\right)=E^{\prime}\left(\Delta t_{L}^{\prime}\right) \frac{1+\Delta t_{L} /\left(\kappa_{\mathrm{F}} \tau_{\mathrm{F}}\right)}{1+\Delta t_{L}^{\prime} /\left(\kappa_{\mathrm{F}} \tau_{\mathrm{F}}\right)}
$$

Outside the linear region $20 \tau_{\mathrm{FC}}<\Delta t_{L}<\tau_{\text {or }} / 2$ the true energy value is obtained by multiplying the result of Equation 12 with a correction factor $F_{E}$ which is diagrammed in Fig. 3a.

\section{Calibration curves}

Energy calibration curves $T_{E}\left(E^{\prime}\right)$ and pulse duration calibration curves $T_{E}\left(I_{0 L}^{\prime}\right)$ are presented in Figs. 5 and 6, respectively. A Gaussian temporal pulse shape is assumed. The spatial pulse shape is either rectangular within a circular aperture of radius $r_{0}$ (Figs. 5a, 6a) or Gaussian (Figs. 5b, 6b). Curves are calculated for the fundamental and second harmonic of the ruby laser (Curves $1^{\prime}$ and $2^{\prime}, \Delta t_{L}^{\prime}=25 \mathrm{ps}$ ) as well as for the fundamental, second, third and fourth harmonic of the Nd-glass laser (Curves 2, $1,3,4, \Delta t_{L}^{\prime}=6 \mathrm{ps}$ ). The curves are calculated for $\tau_{\mathrm{FC}}=0$ and $\tau_{\mathrm{or}}=\infty$ (Equations 7-10) with a small signal dye transmission of $T_{0}=0.01$. The other applied pulse and dye parameters are listed in Table I.

The pulse durations are obtained by measuring $T_{E}$ and $I_{0 L}$ and reading $I_{0 L}^{\prime}\left(T_{E}\right)$ from Fig. 5. Then Equation 11 is applied to obtain the apparent duration $\Delta t_{L, a}$. Finally the correction factors of Fig. $3 \mathrm{~b}$ are used to calculate $\Delta t_{L}=F_{t}\left(\Delta t_{L, a} / \tau_{\mathrm{FC}}\right) F_{t}\left(\Delta t_{L, a} / \tau_{0 r}\right) \Delta t_{L, a}$. Similarly, the pulse energy densities are determined by measuring $T_{E}$ and reading the corresponding $E^{\prime} /\left(\pi r_{0}^{2}\right)$-value from Fig. 6. Equation 12 is used to correct for different pulse durations between the experiment and calibration curve. For pulse 

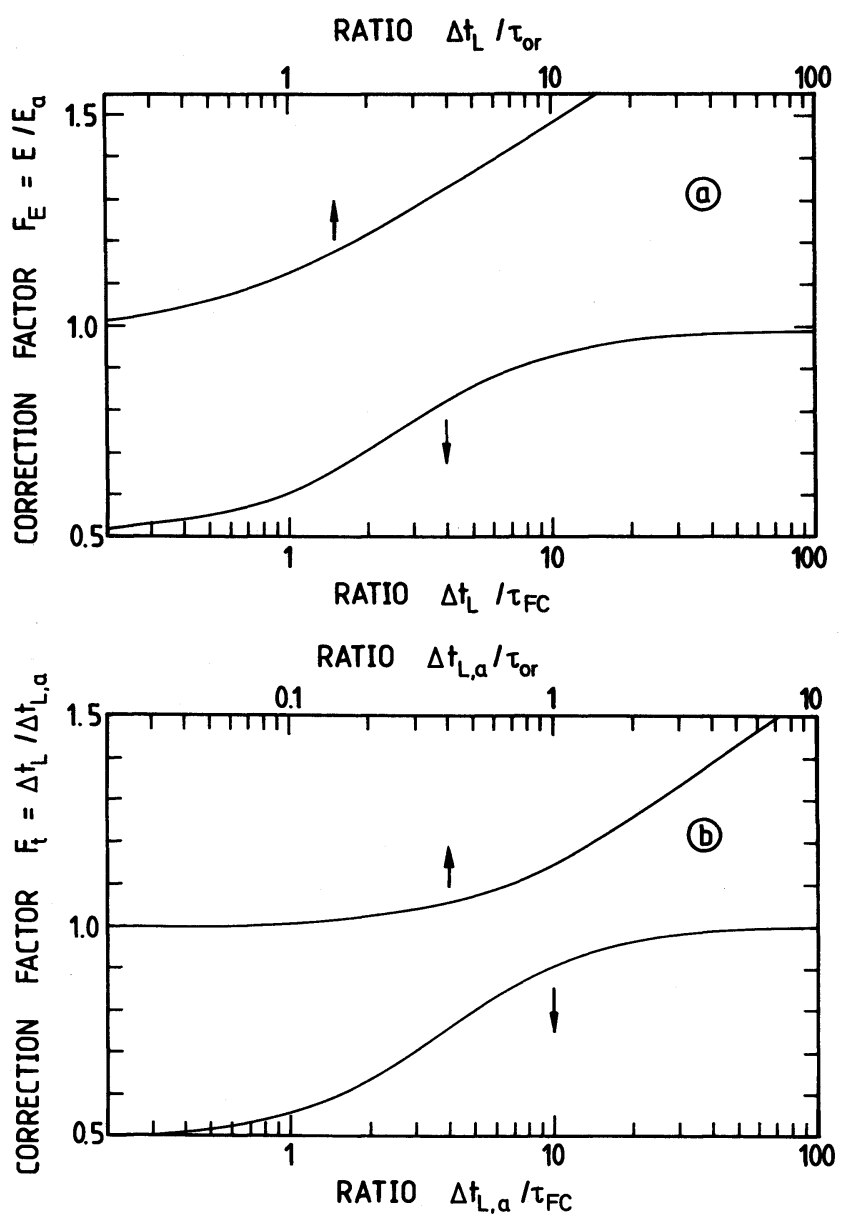

Figure 3 (a) Correction factors for true energy determination, $E$, from apparent values $E_{a}$ obtained for $\tau_{\mathrm{FC}}=0$ and $\tau_{0 r}=\infty$ (Equation 12). (b) Correction factors for determination of true pulse durations $\Delta t_{L}$ from apparent values $\Delta t_{L, a}$ (Equation 11).

TA B LE I Parameters of applied dyes

\begin{tabular}{|c|c|c|c|c|c|c|c|}
\hline Laser & $\begin{array}{l}\text { Wavelength } \\
(\mathrm{nm})\end{array}$ & Dye & Solvent & $\begin{array}{l}\sigma_{L} \\
{\left[10^{-16} \mathrm{~cm}^{2}\right]}\end{array}$ & $\begin{array}{l}\sigma_{\mathrm{ex}} \\
{\left[10^{-17} \mathrm{~cm}^{2}\right]}\end{array}$ & $\begin{array}{l}\tau_{\mathrm{F}} \\
{[\mathrm{ns}]}\end{array}$ & $\begin{array}{r}-\tau_{0} r \\
{[\mathrm{~ns}]}\end{array}$ \\
\hline Ruby & 694.3 & $\mathrm{DDI}^{1}$ & glycerol & $4.4 \pm 0.2$ & $4.8 \pm 0.5$ & $0.26^{5}$ & $1.0^{5}$ \\
\hline Ruby, SH & 347.15 & Dimethyl POPOP & cyclohexane & $1.74^{3}$ & $4.9 \pm 0.5$ & $1.5^{3}$ & $0.2^{7}$ \\
\hline Nd-glass & 1055 & $\mathrm{BDN} \mathrm{I}^{2}$ & 1,2-dichloroethane & $1.07 \pm 0.05$ & 1.9 & $1^{6}$ & $0.28^{7}$ \\
\hline Nd-glass, SH & 527.5 & Rhodamine $6 \mathrm{G}$ & ethanol & $4.17^{4}$ & $5^{4}$ & $4.2^{4}$ & $0.3^{8}$ \\
\hline Nd-glass, TH & 351.7 & Dimethyl POPOP & cyclohexane & $1.8^{3}$ & $4.9 \pm 0.5$ & $1.5^{3}$ & $0.2^{7}$ \\
\hline Nd-glass, FH & 263.8 & 9,10-dimethylanthracene & cyclohexane & $3.3 \pm 0.2^{3}$ & $12.5 \pm 1$ & $6.1^{3}$ & $0.11^{7}$ \\
\hline
\end{tabular}

${ }^{1}$ 1,1'-diethyl-2,2'-dicarbocyanine iodide (Kodak dye No. 9618).

${ }^{2}$ Bis-(4-dimethylaminodithiobenzil)nickel (Eastman dye No. 14015).

${ }^{3}$ From Berlman [8].

${ }^{4}$ From Falkenstein et al. [4].

${ }^{5}$ From Seymour et al. [9].

${ }^{6}$ From Drexhage et al. [10].

${ }^{7}$ Estimated from Debye-Stokes-Einstein hydrodynamic model [11, 12].

${ }^{8}$ From Lessing et al. [13]. 


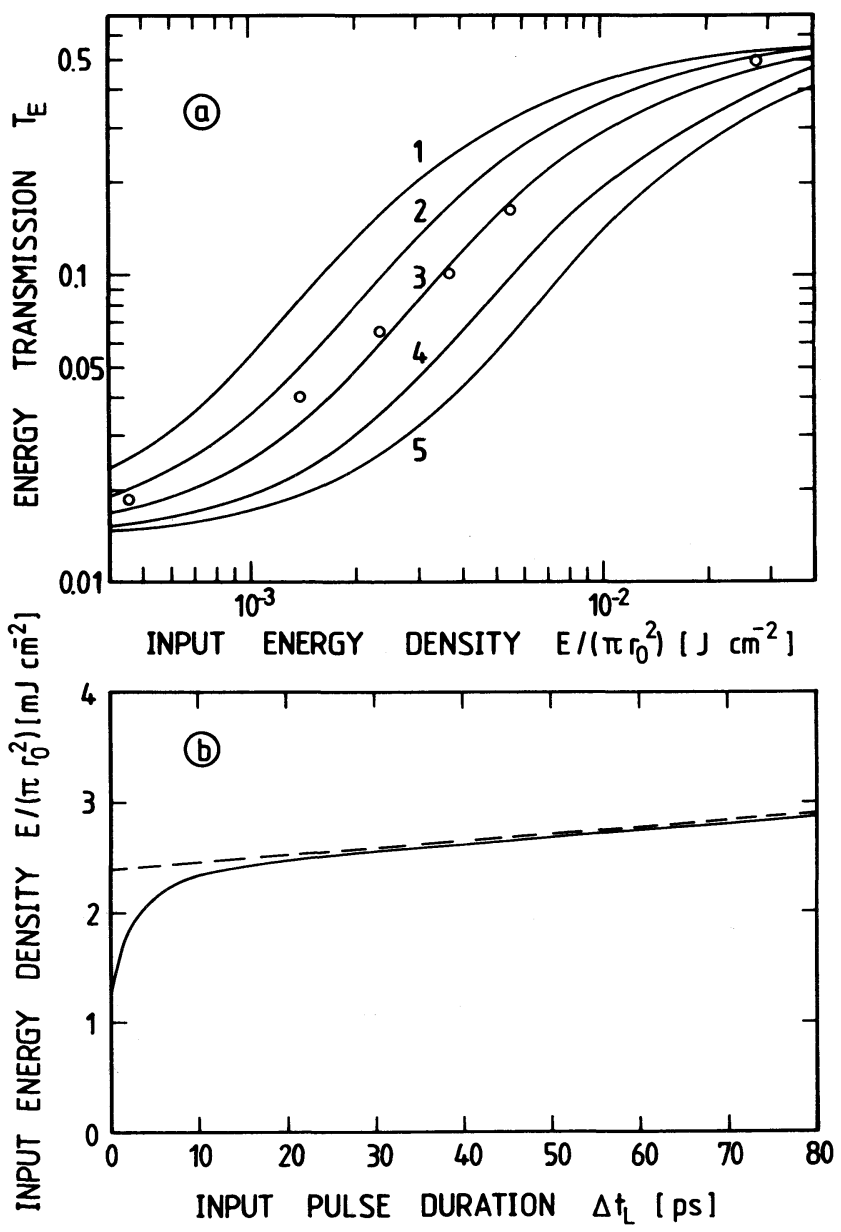

Figure 4 (a) Energy transmission against normalized input pulse energy. Dye and pulse parameters as in Fig. 2a. Pulse durations $\Delta t_{L}$ are (1) $0 \mathrm{ps,} \mathrm{(2)} 2 \mathrm{ps,} \mathrm{(3)} 50 \mathrm{ps,}$ (4) 260 ps, (5) 520 ps. Circles are experimental points for $\Delta t_{L}=30 \pm 10 \mathrm{ps}$. (b) Normalized input pulse energy against input pulse duration at fixed energy transmission of $T_{E}=0.07$. Solid curve, data as in Fig. 4a (Equations 1-6). Dashed curve, linearized solution (Equations 7-10).

durations outside the linear region the apparent pulse energy (Equation 12) is multiplied by the correction factors of Fig. 3a.

\section{Experiments}

The dye parameters entering the calculations are taken from the literature where possible (fluorescence lifetimes and reorientation times). The ground state absorption cross-sections are measured with a spectrophotometer. The excited state absorption cross-sections are obtained from energy transmission measurements at high input laser intensities ( $\sigma_{\text {ex }}$ limits bleaching at high pump intensities).

For the mode-locked ruby laser the calculated curves are compared with measurements of energy and pulse duration. The energy was measured with a pyroelectric detector (Gen tec ED 100). The beam radius $r_{0}$ was determined with a silicon diode array (Tracor DARSS system). The energy transmission through the dye solutions was measured with two photodetectors and a fast transient digitizer (Tektronix R7 912). The obtained $T_{E}$ values against the measured normalized energy $E /\left(\pi r_{0}^{2}\right)$ are shown in Fig. 4a by the data points (mean pulse duration $30 \mathrm{ps).} \mathrm{The} \mathrm{experimental} \mathrm{points} \mathrm{agree} \mathrm{with}$ the calculation within $\pm 10 \%$. Fast photodetectors were calibrated to absolute energy detection by energy transmission measurement with spatial truncated Gaussian pulses. For aperture radii $r_{\boldsymbol{A}} \lesssim 0.5 r_{0}$ the calibration curves for rectangular spatial shape (Fig. 6a) are good approximations.

The pulse durations were measured by the two-photon fluorescence technique [14] (dye: $2.5 \times 10^{-3}$ molar rhodamine $6 \mathrm{G}$ in ethanol). The input peak intensity is obtained from nonlinear transmission 


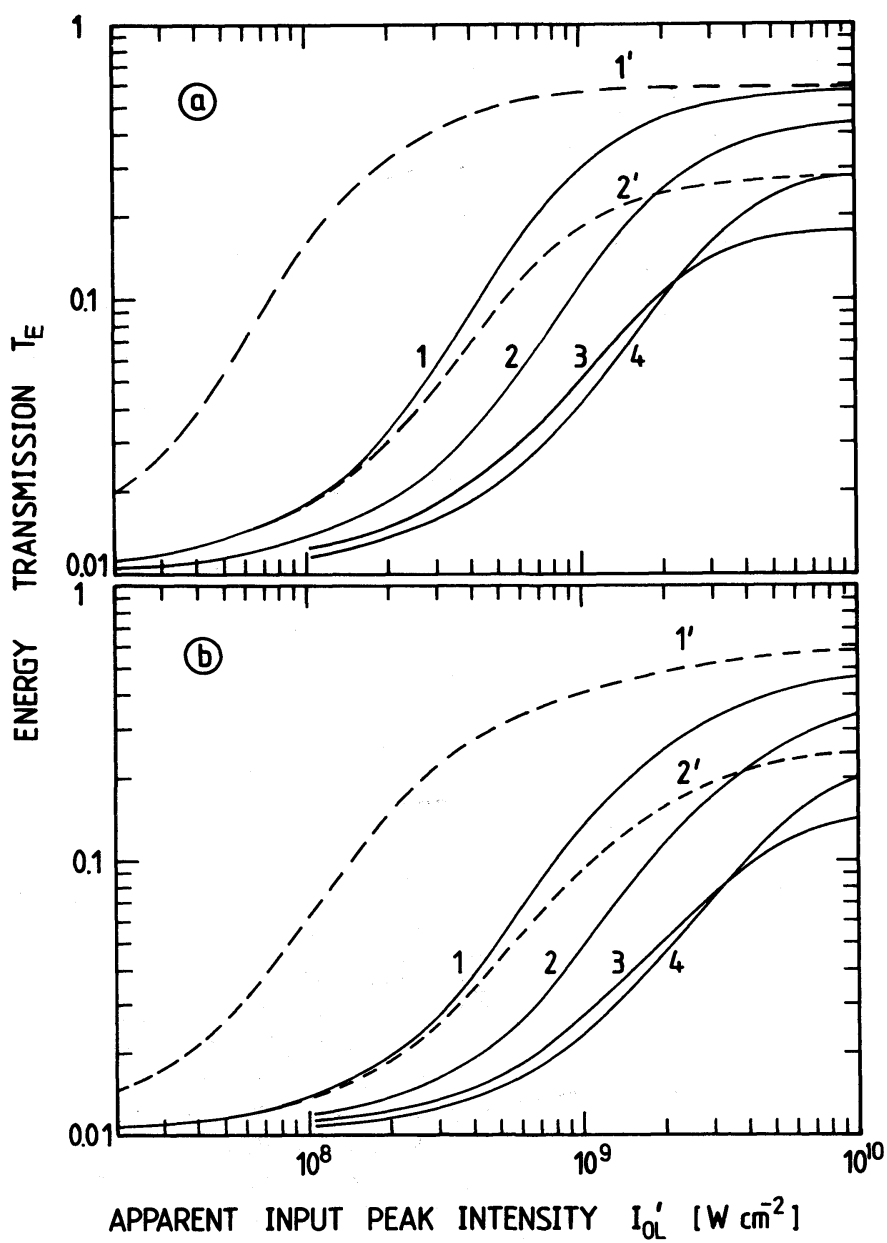

Figure 5 Calibration curves for pulse duration determination. Temporal pulse shape is Gaussian; spatial pulse shape is rectangular in (a) and Gaussian in (b). Dashed curves: $\left(1^{\prime}\right)$ fundamental ruby laser, (2') second harmonic of ruby laser. Solid curves: (1) second harmonic, (2) fundamental, (3) third, (4) fourth harmonic of $\mathrm{Nd}$-glass laser. Small signal dye transmission $T_{0}=0.01$. Other data are listed in Table 1 .

measurements through a two-photon absorbing CdS crystal [15] (the two-photon absorption crosssection of Blau and Penzkofer [15] corrected to $\left.\alpha^{(2)}=1.3 \times 10^{-8} \mathrm{~cm} \mathrm{~W}^{-1}\right)$. Measured energy transmission points against input peak intensity are included in Fig. 2 a for pulses with measured duration of $30 \pm 3$ ps. The experimental points agree with calculation within $\pm 25 \%$. The accuracy is limited by the accuracy of the measurement of photodetector signals for $T_{E}$ and $I_{0 L}$ determination.

Two photon-absorption techniques are available for the measurement of the input peak intensity of the ruby laser and its second harmonic [15] and of the second [16], third [16] and fourth [17] harmonic of the $\mathrm{Nd}$-glass laser. A saturable absorber technique may be used for the intensity detection at the fundamental frequency of the $\mathrm{Nd}$-glass laser [18].

\section{Approximate analytical description}

The following estimates may be used for an approximate analytical determination of the energy density of a picosecond pulse.

The absorption of the dye is assumed to be isotropic $\left(\tau_{\text {or }}=0\right)$. The fluorescence lifetime should be long compared to the pulse duration $\left(\tau_{\mathrm{F}} / \Delta t_{L} \rightarrow \infty\right)$. For dyes without excited state absorption the energy density transmission is $T_{\epsilon}=\exp \left[-\sigma_{L}\left(N_{0} l-\epsilon_{\mathrm{Abs}} / h \nu_{L}\right)\right]=T_{0} \exp \left[\sigma_{L} \epsilon\left(1-T_{\epsilon}\right) / h \nu_{L}\right] . l$ is the sample length, $\epsilon_{\mathrm{Abs}}=\epsilon\left(1-T_{\epsilon}\right)$ is the absorbed pulse energy density. Solving the above equation gives

$$
\epsilon=\frac{\ln \left(T_{\epsilon}\right)-\ln \left(T_{0}\right)}{1-T_{\epsilon}} \epsilon_{s}
$$




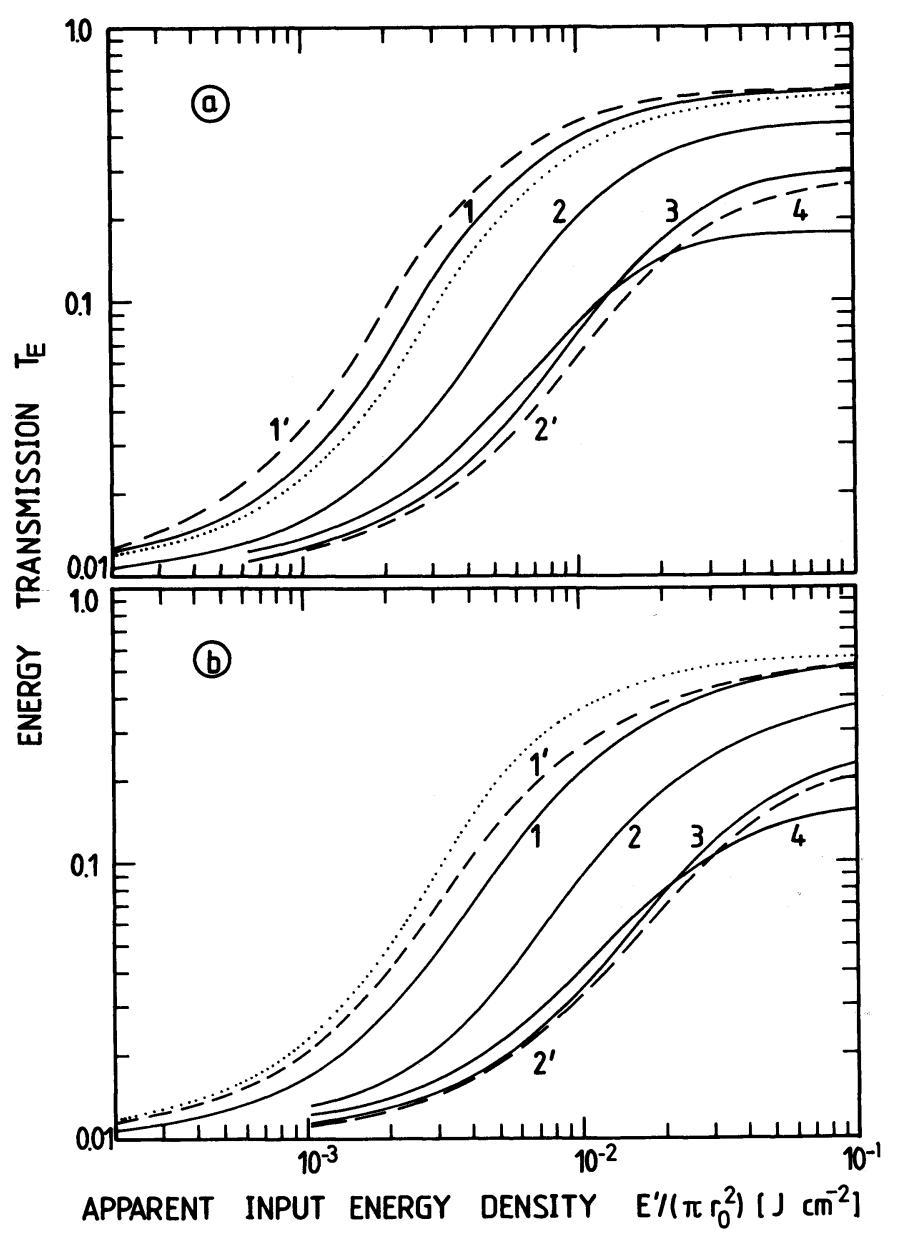

Figure 6 Calibration curves for energy density determination. Temporal pulse shape is Gaussian; spatial pulse shape is rectangular in (a) and Gaussian in (b). Dashed curves: (1') fundamental, (2') second harmonic of ruby laser. Solid curves: (1) second, (2) first, (3) third, (4) fourth harmonic of $\mathrm{Nd}$-glass laser. Dotted curves: analytical solution of Equation 15 for fundamental ruby laser frequency. Small signal dye transmission $T_{0}=0.01$. Other data listed in Table $\mathrm{I}$.

The abbreviation $\epsilon_{s}=h \nu_{L} / \sigma_{L}$ is called the saturation energy density. For finite values of $\Delta t_{L} / \tau_{\mathrm{F}}$ Equation 13 may be extended to

$$
\epsilon=\frac{\ln \left(T_{\epsilon}\right)-\ln \left(T_{0}\right)}{1-T_{\epsilon}} \epsilon_{s}\left(1+\frac{\Delta t_{L}}{\kappa_{F} K_{F}}\right)
$$

Excited state absorption may be included for fast higher excited state relaxation $\left(\tau_{\mathbf{e x}}=0\right)$ in an approximate manner by replacing the denominator $1-T_{\epsilon}$ by $\exp \left(-N_{0} \sigma_{\mathrm{ex}} l\right)-T_{\epsilon}=T_{0}^{\sigma} \mathrm{ex}^{\prime \sigma} L-T_{\epsilon}$ leading to

$$
\epsilon=\frac{\ln \left(T_{\epsilon}\right)-\ln \left(T_{0}\right)}{T_{0}^{\sigma} e^{\prime / \sigma_{L}}-T_{\epsilon}} \epsilon_{s}\left(1+\frac{\Delta t_{L}}{\kappa_{\mathrm{F}} \kappa_{\mathrm{F}}}\right)
$$

The dotted curves in Figs. 6a, b are calculated using Equation 15 for DDI dissolved in glycerol (fundamental ruby laser). They deviate from the corresponding dashed curves 1 ' by a factor of approximately 1.5 (rectangular spatial distribution shown in Fig. 6a) and 0.8 (Gaussian spatial distribution in Fig. 6b).

The deviation from Curve 1' of Fig. 6a results mainly from the fact that anisotropic absorption is not included in Equation $15\left(\tau_{\text {or }}=0\right)$ and slightly from the assumption of infinitely fast relaxation of higher excited states $\left(\tau_{\text {ex }}=0\right)$. In Fig. $6 \mathrm{~b}$ the dotted curve deviates from Curve $1^{\prime}$ since it is not integrated spatially.

The analytical solutions may also be used for the determination of pulse durations by using the fixed dependence of energy on intensity, duration and beam diameter for a specific temporal and spatial pulse shape. 


\section{Conclusions}

For pulse durations short compared to the ground state recovery time, absorbing dyes act as photon counters (absorbed photons = excited photons) and the input pulase energy may be determined from energy transmission measurements. This technique of energy measurement allows one to calibrate photodetectors to absolute picosecond pulse energy. Simultaneous measurements of energy transmission and input peak intensity provides a simple and inexpensive technique to measure the duration of picosecond pulses if they are shorter than the fluorescence lifetime $\left(\Delta t_{L}<\tau_{F} / 2\right)$.

\section{Acknowledgements}

The authors are grateful to Dr W. Blau for many helpful discussions and to Th. Ascherl for technical assistance. They thank the Rechenzentrum of the University for disposal of computer time.

\section{References}

1. A. PENZKOFER and W. BLAU, Opt. Quantum Electron. 15 (1983) 325.

2. A. PENZKOFER, W. FAlKENSTEIN and W. KAISER, Chem. Phys. Lett. 44 (1976) 82.

3. D. REISER and A. LAUBEREAU, Appl. Phys. B27 (1982) 115.

4. W. FAlKENSTEIN, A. PENZKOFER and W. KAISER, Opt. Commun. 27 (1978) 151.

5. A. PENZKOFER and W. FALKENSTEIN, Opt. Quantum Electron. 10 (1978) 399.

6. C. V. SHAN K, E. P. IPPEN and O. TESCHKE, Chem. Phys. Lett. 45 (1977) 291.

7. A. PENZKOFER and J. WIEDMANN, Opt. Commun. 35 (1980) 81.

8. I. B. BERLMAN, 'Handbook of Fluorescence Spectra of Aromatic Molecules' (Academic Press, New York, 1971).

9. R. J. SEYMOUR, P. Y. LEE and R. R. ALFANO, in 'Picosecond Phenomena II' (edited by R. M. Hochstrasser, W. Kaiser and C. V. Shank) Springer Series in Chemical Physics Vol. 14 (Springer, Berlin, 1980) pp. 111-4.

10. K. H. DREXHAGE and U. T. MÜLLER-WESTERHOFF, IEEE J. Quantum Electron. QE-8 (1972) 759.

11. P. DEBYE, 'Polar Molecules' (Dover Publications, London, 1929) p. 84.

12. T. J. CHUANG and K. B. EISENTHAL, Chem. Phys. Lett. 11 (1971) 368.

13. H. E. LESSING and A. VON JENA, in 'Laser Handbook', Vol. 3 (edited by M. L. Stitch) (North Holland, Amsterdam, 1979) Ch. B6, pp. 753-846.

14. J. A. GIORDMAINE, P. M. RENTZEPIS, S. L. SHAPIRO and K. W. WECHT, Appl. Phys. Lett. 11 (1967) 216.

15. W. BLAU and A. PENZKOFER, Opt. Commun. 36 (1981) 419.

16. A. PENZKOFER and W. FALKENSTEIN, ibid. 17 (1976) 1.

17. P. LIU, W. L. SMITH, H. LOTEM, J. H. BECHTEL, N. BLOEMBERGEN and R. S. ADHAV, Phys. Rev. B17 (1978) 4620 .

18. A. PENZKOFER, D. VON DER LiNde and A. LAUBEREAU, Opt. Commun. 4 (1972) 377. 